This item was submitted to Loughborough's Research Repository by the author.

Items in Figshare are protected by copyright, with all rights reserved, unless otherwise indicated.

\title{
Photo-polymerisable electrospun fibres of N-methacrylate glycol chitosan for biomedical applications
}

PLEASE CITE THE PUBLISHED VERSION

http://dx.doi.org/10.1039/c5ra02301g

\section{PUBLISHER}

(c) Royal Society of Chemistry

\section{VERSION}

AM (Accepted Manuscript)

\section{PUBLISHER STATEMENT}

This work is made available according to the conditions of the Creative Commons Attribution-NonCommercialNoDerivatives 4.0 International (CC BY-NC-ND 4.0) licence. Full details of this licence are available at: https://creativecommons.org/licenses/by-nc-nd/4.0/

\section{LICENCE}

CC BY-NC-ND 4.0

\section{REPOSITORY RECORD}

Romano, Ilaria, Elisa Mele, Jose A. Heredia-Guerrero, Luca Ceseracciu, Hadi Hajiali, Luca Goldoni, Lara Marini, and Athanassia Athanassiou. 2015. "Photo-polymerisable Electrospun Fibres of N-methacrylate Glycol Chitosan for Biomedical Applications”. figshare. https://hdl.handle.net/2134/17574. 


\title{
Photo-polymerisable electrospun fibres of $N$-methacrylate glycol chitosan for biomedical applications
}

\author{
Ilaria Romano, ${ }^{* a}$ Elisa Mele, ${ }^{* a, b}$ José A. Heredia-Guerrero, ${ }^{a}$ Luca Ceseracciu, ${ }^{a}$ Hadi Hajiali, ${ }^{a, c}$ Luca \\ Goldoni, ${ }^{d}$ Lara Marini, ${ }^{a}$ Athanassia Athanassiou ${ }^{*}{ }^{*}$ \\ 5* E-mail: ilaria.romano@iit.it; elisa.mele@iit.it; athanassia.athanassiou@iit.it
}

\author{
Received (in $X X X, X X X) X$ th $X X X X X X X X X 20 X X$, Accepted Xth $X X X X X X X X X 20 X X$ \\ DOI: 10.1039/b000000x
}

\begin{abstract}
The availability of nanofibrous substrates with engineered properties, such as controlled 10 porosity, mechanical conformability, biodegradation profile and drug release, is of strategic importance in the biomedical sector. Here, we demonstrate that N-methacrylate glycol chitosan, a photo-polymerisable, biocompatible and water-soluble derivative of chitosan, can be easily processed to create non-woven mats of nanofibres with controlled physicochemical characteristics. The produced fibrous mats are characterised by thermal stability and by Young's modulus and ultimate strength of about $140 \mathrm{MPa}$ and 4 ${ }_{15} \mathrm{MPa}$, respectively. The degree of cross-linking of the realised fibres was important for the regulation of their durability and degradation profile under conditions of high humidity, but also for the controlled delivery over time of antimicrobial drugs encapsulated inside the fibres. We demonstrated that the Nmethacrylate glycol chitosan nanofibres were able to release the active agent within 24 hours, which is a typical time for dressing change and wound inspection. Moreover, cell proliferation of $85 \%$ was 20 measured, indicating that non-cytotoxic substances were released from the electrospun fibres.
\end{abstract}

\section{Introduction}

Scaffolding materials to be used in regenerative medicine and tissue engineering require characteristics of excellent biocompatibility, controlled biodegradability and engineered ${ }_{25}$ porosity. ${ }^{1-6}$ It is of paramount importance that the degradation time of these substrates is comparable to the time needed for the healing and/or the regeneration of the target tissue. Moreover, architectures with appropriate permeability are needed to promote the diffusion of nutrients, the release of drugs, and the 30 proliferation of cells.

Photopolymerisable polymers derived from naturally-occurring polysaccharides represent an interesting class of biomaterials, whose biodegradation profile can be easily tuned by acting on their degree of crosslinking. ${ }^{7-9}$ To this purpose, chemically 35 modified chitosan, a polymer obtained from the exoskeleton of arthropods or from the cell walls of fungi, ${ }^{10}$ has been extensively exploited. In particular, Amsden and co-workers synthesised a water soluble derivative of chitosan, $N$-methacrylate glycol chitosan (MGC), by modifying glycol chitosan with glycidyl 40 methacrylate. ${ }^{11}$ MGC can be cross-linked by ultraviolet (UV) light, and its use has been demonstrated for cell encapsulation and drug delivery. ${ }^{12-16}$ One important property of the MGC hydrogel is the non-cytotoxicy that was advantageously exploited in the realisation of injectable scaffolds for the regeneration of 45 soft tissues. ${ }^{17-19}$

To the best of our knowledge, so far MGC has been only processed as bulk un-textured hydrogel, but not in the form of nanofibres. The availability of MGC nanofibrous substrates could significantly increase the potentialities of this photopolymerisable 50 material in the biomedical sectors. In fact, non-woven mats of nanofibres, realised by the electrospinning (ES) technique, possess high specific surface area, interconnected porosity and mechanical conformability. These features are essential in tissue regeneration for promoting the cells growth since they mimic the 55 extracellular matrix (EMC) architecture, they can guarantee efficient transport of nutrients and gases to the cells, but also controlled release of loaded bioactive compounds. ${ }^{20-22}$

Here, we show for the first time the fabrication of non-woven mats of long and well-defined nanofibres of $N$-methacrylate ${ }_{60}$ glycol chitosan by electrospinning. Acting on the cross-linking degree of the resulting MGC fibrous scaffolds using UV light, we regulated their degradability in moisture-rich atmospheres and effectively controlled the rate of release of encapsulated drugs. We simulated the moist environment of an open wound, and ${ }_{65}$ demonstrated that fibres mats of specific thickness were able to deliver antimicrobial agents in the time frame of 24 hours (typical time of replacement of a wound bandage), while their integrity was preserved in order to be easily managed and removed. Furthermore, the high flexibility offered by the here presented 70 electrospun MGC supports makes them promising as biomedical devices. 


\section{Experimental}

\section{Chemicals and cell line}

Glycol Chitosan, Dimethylsulfoxide (DMSO), the photoinitiator Irgacure 2959, Glycidyl Methacrylate, Polyethylene oxide (PEO, $5 \mathrm{Mw} 600000 \mathrm{~g} / \mathrm{mol}$ ), Sodium hydroxide, Hydrochloric acid, Agarose, Deuterium oxide (DO), Sodium deuteroxide (NaOD), Dulbecco's Modified Eagle's Medium (DMEM), Bovine Calf Serum (BCS), Trypsin-EDTA solution, Penicillin-Streptomycin and Thiazolyl Blue Tetrazolium Bromide (MTT) were purchased

10 from Sigma Aldrich. A water solution of eosin at $2 \%$, known as Neomercurocromo ${ }^{\circledR}$, was obtained from Laboratorio Farmaceutiche Specialità Igienico Terapeutiche S.I.T. s.r.l. Fibroblast cells from murine embryo (NIH3T3-cells) were obtained from ATCC® (CRL-1658TM).

15

\section{Synthesis and characterisation of MGC}

$\mathrm{N}$-methacrylate glycol chitosan was synthesised according to the procedure described in Ref. 15. Briefly, the commercially available glycol chitosan was first purified by $50 \mathrm{kDa}$ dialysis

20 membrane against water for 48 hours. Then, an aqueous solution containing $1.9 \% \mathrm{w} / \mathrm{v}$ of the purified glycol chitosan was prepared, and adjusted with $\mathrm{NaOH} 1 \mathrm{M}$ to reach $\mathrm{pH}$ 9.0. The solution was then reacted with glycidyl methacrylate for 24 hours to obtain a $3 \%$ degree of substitution (DOS) and neutralised with $\mathrm{HCl} 0.1 \mathrm{M}$.

25 The resulting material was purified by $12 \mathrm{kDa}$ dialysis membrane against water for 8 hours, and lyophilised for 2-3 days. In this way, a powder of N-methacrylate glycol chitosan with a degree of substitution (DOS) of 3\% was obtained, as demonstrated by NMR analysis (Figure S1). ${ }^{1} \mathrm{H}$ NMR spectra were acquired at ${ }_{30} 400.13 \mathrm{MHz}$ with a spectrometer Bruker Avance 400 Ultra Shield TM Plus. The MGC powder was dissolved in DO at a concentration of $20 \mathrm{mg} / \mathrm{mL}$, and the resulting solution was adjusted with $0.1 \mathrm{M} \mathrm{NaOD}$ to reach a $\mathrm{pH}>10$. The samples were allowed to equilibrate for 10 minutes at $90{ }^{\circ} \mathrm{C}$ within the 35 spectrometer prior to shimming. Before each acquisition the automatic matching and tuning was run and the homogeneity was automatically adjusted. 128 transients were accumulated by applying a 30 flip angle, $65 \mathrm{~K}$ complex data points, with a spectral width of $20.5 \mathrm{ppm}$ and a relaxation delay of 5 seconds. The

40 temperature was actively controlled at $363 \mathrm{~K}$. The free induction decay (FID) signals were multiplied by an exponential function equivalent to that of $0.5 \mathrm{~Hz}$ line-broadening factor and then Fourier transformed. Spectra were manually phased and automatically baseline corrected by means of an automatic 45 alternate algorithm (Topspin 3.1, Bruker) and referred to the signal at $6.2 \mathrm{ppm}$, as reported by work presented by Amsden et al. ${ }^{15}$. Each integral was manually adjusted by a bias and a slope correction.

\section{${ }_{50}$ Fabrication of the MGC fibrous mats}

Water solutions consisting of $4 \% \mathrm{w} / \mathrm{v}$ of MGC and 1 or $5 \% \mathrm{w} / \mathrm{v}$ of photoinitiator Irgacure 2959 were prepared and used for electrospinning (ES). To facilitate the formation of the nanofibres, an optimised amount of Polyethylene oxide (PEO)

55 was added to the pristine MGC solution (MGC/PEO relative ratio of 7:3). As previously demonstrated by other groups, the majority of polysaccharides and their derivatives are hard to electrospin. ${ }^{23}$ For this reason, biocompatible polymers, like PEO, are typically used as additives. ${ }^{24,25}$ From now on any reference in the 60 manuscript to MGC nanofibres would imply the blend MGC/PEO (7:3). Moreover, in order to improve the uniformity of the nanofibres, we added DMSO as co-solvent to the initial MCG solutions (concentration of $10 \% \mathrm{w} / \mathrm{v}$ ). DMSO plays a double role that is essential for the improvement of the electrospinning ${ }_{65}$ procedure of MGC. First, due to its high dielectric constant, ${ }^{26,27}$ it increases the electrical conductivity of the MGC solution, and second it loosens the chitosan chain entanglement. ${ }^{25}$

In our ES set-up, the fibres were extruded by applying a voltage of $10 \mathrm{kV}$ (high voltage power supply EH40R2.5, Glassman High 70 Voltage Inc.) between a stainless steel 23-gauge needle and an aluminium plate, placed at a relative distance of $20 \mathrm{~cm}$. The solutions were injected through the needle by a syringe pump (NE-1000, New Era Pump Systems Inc.) working at a flow rate of $0.6 \mathrm{~mL} \mathrm{~h}^{-1}$. Immediately after production (without drying 75 procedures), part of the collected fibres was cross-linked by using UV light at $(405 \pm 10) \mathrm{nm}\left(20 \mathrm{~mW} / \mathrm{cm}^{2}\right.$, irradiation time of 30 minutes).

\section{Characterisation of the nanofibres}

${ }_{80}$ The morphology and the size of the electrospun fibres were analysed by Scanning electron microscopy (SEM). A JEOL JSM6490LA microscope working in high vacuum mode, with an acceleration voltage of $15 \mathrm{kV}$, was used. A coating of $10 \mathrm{~nm}$ $\mathrm{Au} / \mathrm{Pd}$ was required to prevent charging effects. We investigated 85 the chemical properties of the MGC fibres by an attenuated total reflectance (ATR) accessory (MIRacle ATR, PIKE Technologies) coupled to a Fourier transform infrared (FTIR) spectrometer (Equinox 70 FT-IR, Bruker). The spectra were recorded in the range from 3800 to $600 \mathrm{~cm}^{-1}$, with $4 \mathrm{~cm}^{-1}$ 90 resolution (accumulating 128 scans).

The mechanical properties of the scaffolds were characterised by uniaxial tension tests on a dual column universal testing machine (Instron 3365). Mats were cut in prismatic specimen with a width of $4 \mathrm{~mm}$ and an effective length of $25 \mathrm{~mm}$. In order to avoid 95 accidental damage during handling, specimens were first taped on custom made paper frames composed by two detachable halves, then mounted on the measuring machine's hydraulic clamps. Displacement was applied with the rate of $1 \mathrm{~mm} / \mathrm{min}$. From the resulting stress strain curves, the Young's modulus, ultimate 100 strength and elongation at fracture were extracted. Measurements were performed on 5 samples.

The thermal degradation behaviour of the electrospun MGC/PEO nanofibres and of the pure components (MGC and PEO) was investigated by thermogravimetric (TGA) analysis using the ${ }_{105}$ Q500 analyser from TA Instruments. The measurements were performed under an inert $\mathrm{N}_{2}$ atmosphere with a flow rate of 50 $\mathrm{mL} / \mathrm{min}$ in a temperature range from 30 to $600^{\circ} \mathrm{C}$ at heating rate of $10^{\circ} \mathrm{C} / \mathrm{min}$.

The biocompatibility of the fibrous MGC scaffolds was evaluated 110 by indirect cytotoxicity assays with immortalized mouse fibroblasts (NIH/3T3) as model cell line. After UV cross-linking and sterilization, the fibrous mats were immersed in the culture medium (DMEM) supplemented with $10 \%$ BCS and $1 \%$ 
antibiotics $(100 \mathrm{U} / \mathrm{mL}$ penicillin and $0.1 \mathrm{mg} / \mathrm{mL}$ streptomycin) for 24 hours, in order to obtain their complete dissolution. In particular, according with the ISO10993-5 standard test method, a fibrous sample with an area of $6 \mathrm{~cm}^{2}$ was dissolved in $1 \mathrm{~mL}$ of 5 DMEM. Meanwhile, NIH3T3 cells were seeded on a 96-well tissue culture polystyrene plate (TCPS) with a density of about 10000 cells/well, and cultured for 24 hours at $37{ }^{\circ} \mathrm{C}$ with $5 \% \mathrm{CO}_{2}$ to allow the attachment. Then, the culture medium was replaced with the extraction one and the cells were incubated for other 24 10 hours.

\section{Investigation of the fibre stability and drug release in aqueous environments}

The realised fibrous samples $(5 \% \mathrm{w} / \mathrm{v}$ of photoinitiator, thickness $15 \sim 300 \mu \mathrm{m})$ were stored in a box with controlled humidified atmosphere at $100 \%$ relative humidity (temperature of $25{ }^{\circ} \mathrm{C}$ ), and their structural stability was analysed by SEM after fixed time intervals.

As model compound, the antimicrobial Neomercurocromo ${ }^{\circledR}$ was 20 incorporated inside the MGC fibres. A MGC:PEO solution containing $1 \mathrm{v} / \mathrm{v} \%$ of Neomercurocromo was prepared and electrospun according to the procedure previously described. Composite nanofibrous mats (before and after UV irradiation) of area $1 \mathrm{~cm}^{2}$ (thickness $\sim 300 \mu \mathrm{m}$ ) were placed in contact with the ${ }_{25}$ surface of a thick film of agarose hydrogel ( $1 \%$ in water, $\left.\mathrm{pH} 7\right)$. The diffusion of eosin through the hydrogel was recorded as a function of time by using a UV-Visible spectrophotometer, Varian Cary 6000i, in absorbance mode from 200 to $800 \mathrm{~nm}$. In particular, the agarose was previously gelified inside a cuvette, 30 and the samples were gently deposited onto the hydrogel, before positioning the cuvette in the spectrophotometer. Then, the absorption spectra were collected regularly over time, from a portion of the gel located just underneath the fibrous mats. The experiments were performed in quintuplicate at room 35 temperature.

\section{Results and Discussion}

The morphological analysis, carried out by SEM, revealed that the electrospun mats of MGC with $1 \%$ photoinitiator mainly 40 consisted of a dense network of nanofibres (Fig. 1a). However, the fibres' diameter appeared non-uniform throughout their length, since bead-like features were present. As clearly visible in Fig. 1b, the structure of the electrospun fibres was substantially improved by the addition of DMSO, since the presence of beads 45 was strongly reduced. The resulting nanofibres showed cylindrical shape and regular morphology with an average diameter of $(172 \pm 31) \mathrm{nm}$. The nanofibres' size distribution was also quite homogeneous, since $80 \%$ of the population had diameters in the range of 150-210 nm (Fig. 1d). Furthermore, 50 well-defined homogeneous nanofibres were also produced by increasing the amount of photoinitiator from 1 to $5 \%$ (Fig. 1c). In this case, the average diameter increased, becoming (266 \pm 39$)$ $\mathrm{nm}$ (Fig. 1d), and $40 \%$ of the population was monodispersed with a size of $230 \mathrm{~nm}$. On the contrary, when the degree of acrylation 55 of MGC was increased from 3 to $7 \%$, we observed the formation of beaded fibrillar structures (Figure S2).
After production, the fibres were cross-linked using UV light. No appreciable differences were observed in the size and morphology of the nanofibres before and after light exposure. ${ }_{60}$ Indeed, the SEM pictures presented in Figure 1 represent the cross-linked electrospun mats but they are identical to the ones just-electrospun (Figure S3). It is worth noting that, in the case of $\mathrm{ES}$, the cross-linking process is not necessary for imparting a physical shape to the fibres. In fact, during ES complete ${ }_{65}$ evaporation of the solvent occurs and the nanofibres reach the collector in a dry, solid state, obtaining their final structure even before the UV exposure. The photo-cross-linking procedure solely affects their durability when they are exposed to humid environments, as will be discussed later. On the contrary, when 70 MGC solutions are used for the production of bulk hydrogel scaffolds, the light irradiation is essential to structure the polymer in the desired form in order to be usable. ${ }^{19}$

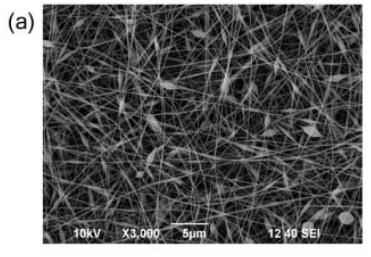

(b)
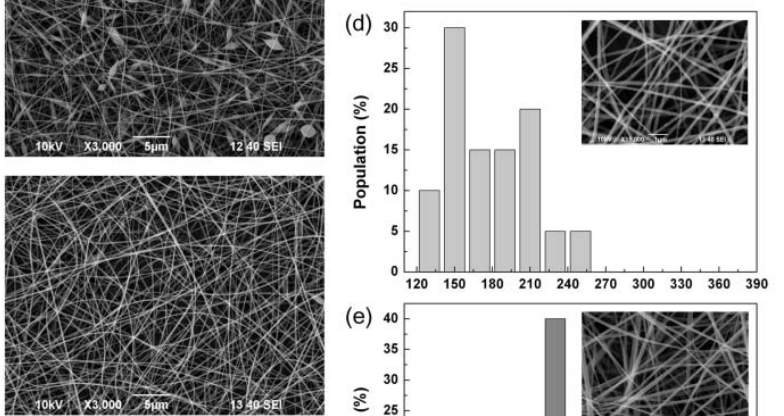

(c)
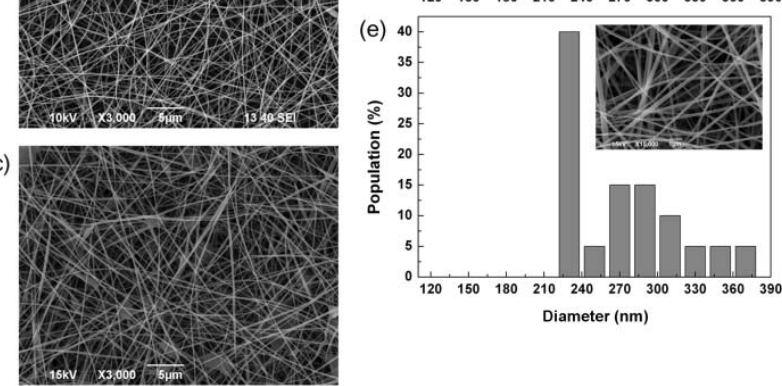

75 Figure 1. SEM images of the cross-linked fibrous networks, electrospun from MGC solutions containing (a) $1 \% \mathrm{w} / \mathrm{v}$ of photoinitiator, (b) $1 \% \mathrm{w} / \mathrm{v}$ of photoinitiator and $10 \% \mathrm{w} / \mathrm{v}$ of DMSO, (c) $5 \% \mathrm{w} / \mathrm{v}$ of photoinitiator and $10 \% \mathrm{w} / \mathrm{v}$ of DMSO. Corresponding size distribution of the realized nanofibres with (d) $1 \%$, and (e) $5 \%$ of photonitiator, and related high 80 magnification SEM pictures in the insets.

As it is possible to observe from the SEM images, the fibres were homogeneously organised within the mats. This was also confirmed by mechanical testing that showed highly reproducible ${ }_{85}$ stress-strain curves (Figure S4). Values of Young's modulus and ultimate strength of $(139 \pm 6) \mathrm{MPa}$ and $(3.7 \pm 0.6) \mathrm{MPa}$ were measured, respectively, with an elongation at fracture of around $5 \%$. Interestingly, the fracture proceeded without progressive inter-fibre shearing and tearing, indicating robustness and a well90 connected structure of the mat. The reported values are comparable with those of chitosan electrospun mats that were cross-linked with other agents, such as glutaraldehyde (GA) vapour or epichlorohydrin. ${ }^{28}$ For instance, Schiffman and coworkers reported values of approximaterily 150 and $1 \mathrm{MPa}$ for 95 the Young's modulus and the tensile strength of GA-crosslinked 
chitosan mats, respectively. ${ }^{29}$ Therefore, MGC can be considered alternative to chitosan in the production of mechanically robust electrospun mats, particularly for biomedical applications that required the use of water soluble compounds.

5 The chemical properties of the electrospun samples were analysed by FTIR. As shown in Fig. 2a, in both just-electrospun and cross-linked nanofibres, the following bands associated to PEO are easily distinguishable: $\mathrm{C}-\mathrm{H}$ stretching at $2880 \mathrm{~cm}^{-1}, \mathrm{CH}_{2}$ scissoring at $1466 \mathrm{~cm}^{-1}, \mathrm{CH}_{2}$ wagging at 1360 and $1340 \mathrm{~cm}^{-1}$, ${ }_{10} \mathrm{CH}_{2}$ twisting at $1279 \mathrm{~cm}^{-1}$, C-O-C stretching at $1095 \mathrm{~cm}^{-1}, \mathrm{CH}_{2}$ rocking at 960 and $841 \mathrm{~cm}^{-1} \cdot{ }^{25}$ Moreover, the following bands ascribed to glycol chitosan can be assigned: the overlap of $\mathrm{O}-\mathrm{H}$ and $\mathrm{N}-\mathrm{H}$ stretching vibrations at $3365 \mathrm{~cm}^{-1}$, the carbonyl $\mathrm{C}=\mathrm{O}-$ NHR stretching at $1670 \mathrm{~cm}^{-1}$ and the bending of non-acylated 215 amineglucose primary amines groups at $1595 \mathrm{~cm}^{-1} \cdot{ }^{30,31}$ However, the vibrations of $N$-methacrylate residues were hardly observed, probably as consequence of their low amount in the final composition of the fibres. As demonstrated by Amsden and coworkers using nuclear magnetic resonance spectroscopy, the $\mathrm{N}$ 20 methacrylate residues are grafted on the glycol chitosan backbone via amine groups. ${ }^{32}$ In order to better characterise the chemical differences in the fibres before and after UV irradiation, we subtracted the spectra of the cross-linked nanofibres from the one of the just-electrospun nanofibres (Fig. $2 \mathrm{~b}$ and 2c). Positive 25 absorptions were observed around $3365\left(v_{\mathrm{O}-\mathrm{H}}\right.$ or $\left.v_{\mathrm{N}-\mathrm{H}}\right), 1650$ and $1595 \mathrm{~cm}^{-1}\left(\delta_{\mathrm{N}-\mathrm{H}}\right)$. The vibration at $1650 \mathrm{~cm}^{-1}$ could be related to the stretching of $\mathrm{C}=\mathrm{C}$ from the methacrylate group. ${ }^{32}$ These positive differences in FT-IR signals are indicative of a loss of $\mathrm{OH}, \mathrm{C}=\mathrm{C}$ and amine functional groups after light irradiation. The 30 opening of the double bonds and the reaction with electron-rich groups (hydroxyl and amine functional groups) can be related to the cross-linking of the chitosan polymer chains, as shown in the schematic representation in Fig. 2d.

(a)

(b)
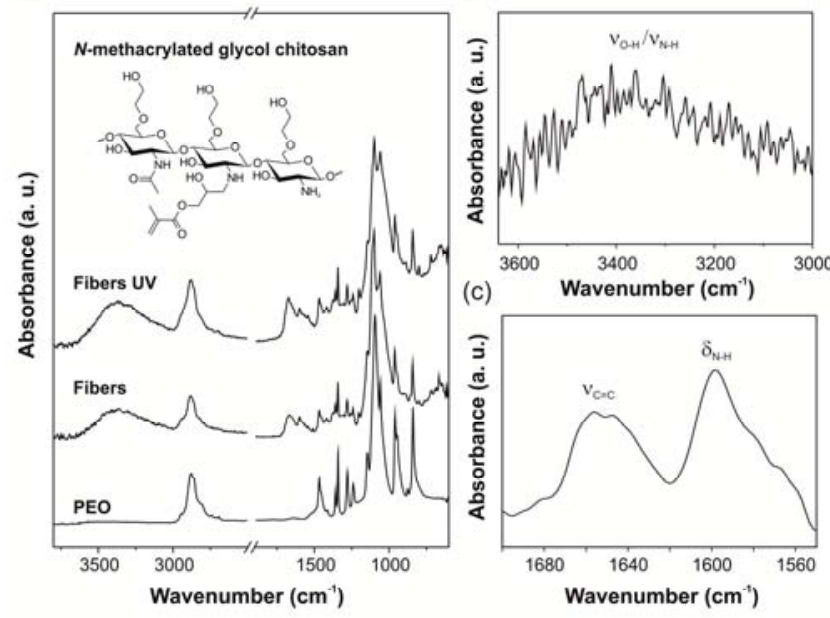

(d)

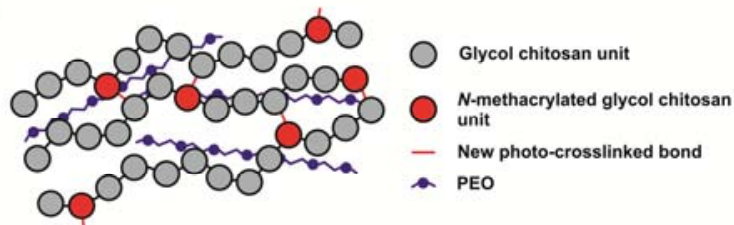

35 Figure 2. (a) ATR-FTIR spectra of PEO and MGC nanofibres containing

$5 \%$ of photoinitiator, before and after UV irradiation. The chemical structure of the $N$-methacrylate glycol chitosan is included in the inset. (b) and (c) Difference between the spectrum of the just-elecrospun and crosslinked fibres in the spectral region of the $3650-3000 \mathrm{~cm}^{-1}$ and $1700-1550$ $40 \mathrm{~cm}^{-1}$, respectively. (d) Schematic representation of the polymer structure of the nanofibres after the UV irradiation.

The structural stability of the MGC electrospun networks, before and after UV exposure, was investigated under specific 45 conditions of humidity. After the observation of the samples at SEM, it is clearly visible that after 1 hour in the moisture-rich environment the not-crosslinked fibres start to fuse together (Fig. $3 a)$, with their diameters getting bigger and the presence of large unstructured regions being visible. This is even more evident $s_{0}$ after 1 day of storage at the high humidity when a complete closure of the pores, previously existing in the electrospun mat, can be observed. Indeed, from Fig. $3 \mathrm{c}$ it is clear that the fibrous network has been totally disappeared, substituted by a uniform MGC film. From the SEM analysis, we estimated that initially, in 55 normal environmental conditions, the $72 \%$ of the sample surface area was occupied by the MGC fibres. Instead, after 1 hour in the humid environment, $90 \%$ of the surface area of the mat was covered by the polymer and the totality after 1 day. On the contrary, the cross-linked fibres were much more stable. In fact, 60 they maintain their shape after 1 hour in the humid atmosphere almost entirely, with only few areas being flattened due to the moisture (Fig. 3b). In fact, in this case image processing showed that the nanofibres were present on the $77 \%$ of the surface area, likewise the as-prepared sample. It is most likely that the high ${ }_{65}$ water solubility of PEO played the main role in the observed topographical changes. After 1 day, the dimensions and the number of the flat regions appear increased $(93 \%$ of the surface area), with a consequent reduction of the porosity of the fibrous network. Nonetheless, the micrometer size pores, typical of the 70 electrospun net, are still visible (Fig. 3d). By comparing the morphology of the just-electrospun and cross-linked MGC nanofibres after their storage at $100 \% \mathrm{RH}$, we can safely state that the cross-linking process allowed us to preserve their integrity of the mat after 24 hours (inset in Fig. 3d). This property

75 is essential for wound managements that require the removal of the dressing after 1 day.

Together with structural stability under humid conditions, the produced MGC-PEO fibrous mats are also thermally stable in a range of temperature between $30-170{ }^{\circ} \mathrm{C}$ (TGA analysis in Fig. ${ }_{80} \mathrm{~S} 5$ ). In fact, they started losing weight at around $200^{\circ} \mathrm{C}$, with two evident degradation steps at around 280 and $400{ }^{\circ} \mathrm{C}$ that are characteristic of pure $\mathrm{MGC}$ and $\mathrm{PEO}$, respectively. Since materials for wound dressing typically operate at temperature lower than $40{ }^{\circ} \mathrm{C}$, we can state that the produced MGC-PEO 85 fibres can be safely applied in this sector without undergoing decomposition.

Moreover, indirect cytotoxicity assays revealed that the produced fibrous mats were biocompatible. To this end, immortalized mouse fibroblasts (NIH3T3) were used as model cell line, 90 following the procedure described above. After cell incubation for 24 hours in the extraction medium (MGC fibres dissolved in DMEM), the adhered and proliferated cells were analysed by a colorimetric MTT assay. As shown in Figure S6, the cell proliferation was of about $85 \%$, indication that the electrospun 95 MGC:PEO fibres were non-cytotoxic and they did not release 
harmful substances to the living cells. Therefore, they can be potentially used for biomedical applications.
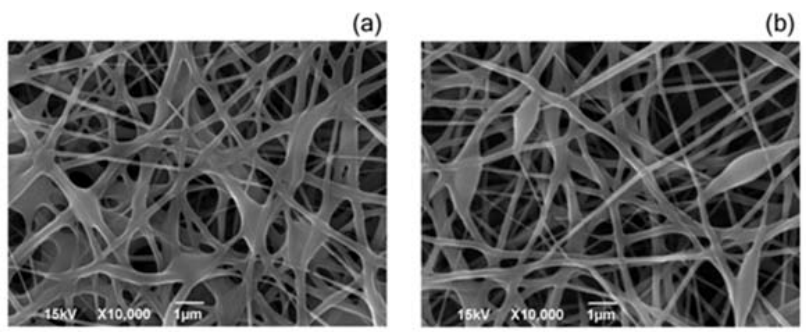

(c)
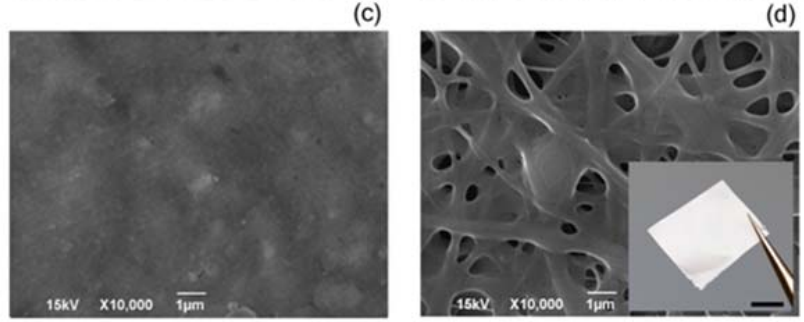

5 Figure 3. SEM images showing the morphology of the electrospun MGC networks exposed to highly humid conditions for different time intervals: after 1 hour at 100\% RH, (a) just-electrospun and (b) cross-linked fibres; after 1 day at $100 \% \mathrm{RH}$, (c) just-electrospun and (d) cross-linked fibres. Inset: photograph of the electrospun mat after 1 day at $100 \% \mathrm{RH}$. Scale

10 bar: $0.5 \mathrm{~cm}$

We observed that by acting on the cross-linking degree of the nanofibres we could control the release rate of active molecules encapsulated inside them. As model compound, a water solution 15 containing $2 \%$ of eosin (Neomercurocromo $\AA$ ) and characterised by antimicrobial activity was incorporated inside the MGC nanofibres. The composite MGC-eosin nanofibrous mats were used for further tests with or without cross-linking. Agarose hydrogel plates were selected as systems to simulate the wet site 20 of an open wound, due to their high water content. ${ }^{33}$ The diffusion of eosin through the hydrogel was directly related to its release from the fibres, and investigated by spectrophotometrical analysis. In Figure 4, the intensity of the characteristic peak of eosin (at $540 \mathrm{~nm}$ ) is plotted for the just-electrospun (black dots)

25 and cross-linked fibres (white dots) at different time intervals. It can be observed that after 1 hour the intensity of the eosin peak increased to about $50 \%$ of its final value for the not cross-linked fibres, whereas it arrived to just $10 \%$ of its final value for the cross-linked ones. Therefore, the amount of eosin released from 30 the not-irradiated samples in short times is much higher (burst release) than that released from the irradiated ones. For the not cross-linked fibrous substrates the measured absorption intensity arrived to its maximum value after 8 hours, indicating total release of the drug from the fibres. On the other hand, in the case 35 of the photopolymerized MGC supports the maximum intensity of the eosin peak was achieved after 1 day. Similar release times have been reported for chitosan electrospun mats containing polyvinyl alcohol ${ }^{34}$ or poly(d,l-lactide-co-glycolide). ${ }^{35}$ In our case, we can also relate the release behaviour of the MGC mats to

40 their degree of solubility when exposed to a moisture-rich environment, as presented above. Indeed, the 24 hours needed for the release of the eosin-compound from the cross-linked nanofibrous mat coincide with the time necessary to the same type of samples to alter their morphology in $100 \% \mathrm{RH}$ ${ }_{45}$ environment but not to totally disintegrate. In order to dispose dressings easier to handle we can further increase the thickness of the UV-treated electrospun fibres, without changing their drug release profile (inset in Fig. 4). This is very useful for daily wound management, when the dressing should be removed and 50 changed after it has released the contained drug in a controlled manner throughout the 24 hours.

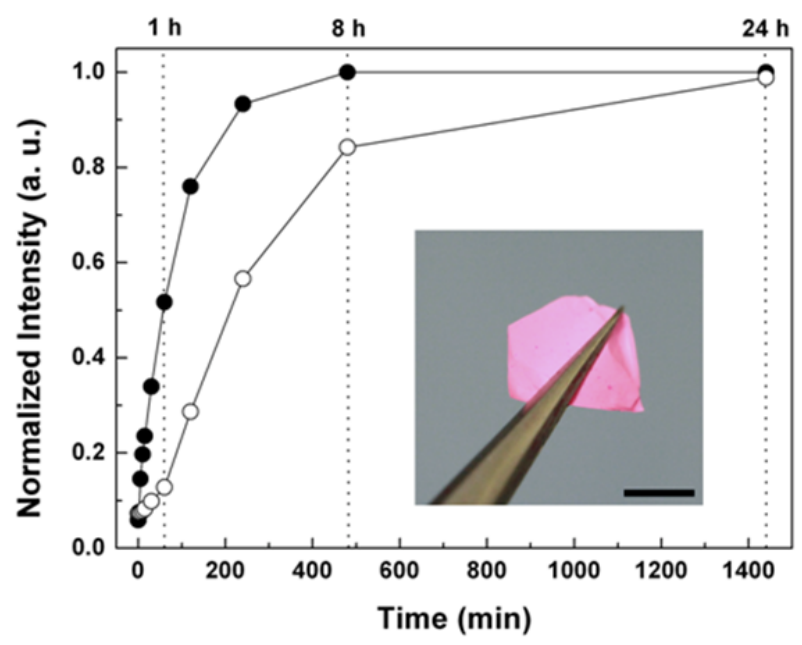

Figure 4. Plot of the maximum intensity values for the absorption peak of eosin released from not- (black dots) and cross-linked (white dots) MGC

55 fibres as function of time. Inset: Photograph of MGC fibrous samples, after UV irradiation, containing $1 \% \mathrm{v} / \mathrm{v}$ of Neomercurocromo. Scale bar: $0.5 \mathrm{~cm}$.

\section{Conclusions}

The effective production of mechanically robust electrospun ${ }_{60}$ nanofibres of a photo-crosslinkable biomaterial, $N$-methacrylate glycol chitosan, was demonstrated. We overcame the difficulties frequently encountered when the ES technique is applied to reticulated polymers and natural polysaccharides, including other chitosan derivatives. Thanks to the process optimization, we were ${ }_{65}$ able to control over time the degradation profile of the fibres and the release rate of the encapsulated hydrophilic drugs. MGC nanofibres can have potential applications in diverse biomedical sectors, including tissue regeneration and wound dressing systems.

\section{${ }_{70}$ Notes and references}

${ }^{a}$ Smart Materials, Nanophysics, Istituto Italiano di Tecnologia, Via Morego 30, 16163, Genoa, Italy.

${ }^{b}$ Present address: Department of Materials, Loughborough University, Loughborough, Leicestershire,LE11 3TU.E-mail: E.Mele3@lboro.ac.uk. $75^{c}$ DIBRIS, University of Genoa, via Opera Pia 13, 16145, Genoa, Italy.

${ }^{d}$ Drug Discovery and Development, Istituto Italiano di Tecnologia, Via Morego 30, 16163, Genoa, Italy.

\section{Acknowledgements}

José A. Heredia-Guerrero is supported by a Marie Curie IntraEuropean Fellowship (BIOPROTO project), financed by the EU's Seventh Framework Programme for Research (FP7). 


\section{REFERENCES}

1 L. S. Nair and C.T. Laurencin Progress in Polymer Science, 2007, 32, 762-798.

52 G. E. Luckachan and C.K.S. Pillai Journal of Polymers and the Environment, 2011, 19, 637-676.

3 G.G. Genchi, G. Ciofani, A. Polini, I. Liakos, D. Iandolo, A. Athanassiou, D. Pisignano, V. Mattoli and A. Menciassi, Journal of Tissue Engineering and Regenerative Medicine, 2012, 1932-6254.

104 S. Beke, F. Anjum, H. Tsushima, L. Ceseracciu, E. Chieregatti, A. Diaspro, A. Athanassiou and F. Brandi, Journal of the Royal Society, Interface, 2012, 9, 3017-3026.

5 F. Brandi, F. Anjum, L. Ceseracciu, A.C. Barone and A. Athanassiou Journal of Micromechanics and Microengineering, 2011, 21, 054007.

6 J.F. Mano, G.A. Silva, H.S. Azevedo, P.B. Malafaya, R.A. Sousa, S.S Silva, L.F. Boesel, J.M. Oliveira, J. M.; T.C. Santos, A.P. Marques, N.M. Neves and R.L. Reis Journal of the Royal Society, Interface, 2007, 4, 999-1030.

207 J.L. Ifkovits and J.A. Burdick, Tissue Engineering, 2007, 13, 2369 2385.

8 O. Jeon, K.H. Bouhadir, J.M. Mansour and E. Alsberg, Biomaterials, 2009, 30, 2724-2734.

9 J.A. Burdick, C. Chung, X. Jia, M.A. Randolph and R. Langer Biomacromolecules, 2005, 6, 386-391.

10 R. Jayakumara, D. Menona, K. Manzoora, S.V. Naira and H. Tamura Carbohydrate Polymers, 2010, 82, 227-232.

11 Z.-M. Huang, Y.-Z. Zhang, M. Kotaki and S. Ramakrishna Composite Science and Technology, 2003, 63, 2223-2253.

3012 J.-P Chen, G.-Y. Chang and J.-K. Chen, Colloids Surfaces A: Physicochemical and Engineering Aspects, 2008, 313-314, 183-188.

13 M. Abrigo, S.L. McArthur and P. Kingshott Macromolecular Bioscience, 2014, 14, 772-792.

14 L. Martinová and D. Lubasová Research Journal of Textile Apparel, 2008, 12, $72-79$

15 B.G. Amsden, A. Sukarto, D.K. Knight and S.N. Shapka, Biomacromolecules, 2007, 8, 3758-3766.

16 J.W.S. Hayami, S.D. Waldman and B.G. Amsden Macromolecular Bioscience, 2011, 11, 1672-1683.

4017 A. Sukarto, C. Yu, L.E. Flynn and B.G. Amsden Biomacromolecules 2012, 13, 2490-2502.

18 J.W.S. Hayami, S.D. Waldman and B.G. Amsden Biomacromolecules, 2013, 14, 4236-4247.

19 H.K. Cheung, T.T.Y. Han, D.M. Marecak, J.F. Watkins, B.G.

$45 \quad$ Amsden and L.E. Flynn Biomaterials, 2014, 35, 1914-1923.

20 M. Ignatova, N. Manolova and I. Rashkov Macromolecular Bioscience 2013, 13, 860-872

21 P.D. Dalton, C. Vaquette, B.L. Farrugia, T.R. Dargaville, T.D. Brown and D.W. Hutmacher Biomaterials Science, 2013, 1, 171-185.

5022 W. Liu, S. Thomopoulos and Y. Xia Advanced Healthcare Materials, 2012, 1, 10-25.

23 A.C. Stijnman, I. Bodnar and R.H. Tromp, Food Hydrocolloids, 2011, 25, 1393-1398.

24 C.A. Bonino, K. Efimenko, S.I. Jeong, M.D. Krebs, E. Alsberg and S.A. Khan Small, 2012, 8, 1928-1936.

25 N. Bhattarai, D. Edmondson, O. Veiseh, F.A. Matsen and M. Zhang, Biomaterials, 2005, 26, 6176-6184.

26 N. Choktaweesap, K. Arayanarakul, D. Aht-Ong, C. Meechaisue and P. Supaphol, Polymer Journal, 2007, 39, 622-631.

6027 H. Jiang, D. Fang, B.S. Hsiao, B. Chu and W. Chen Biomacromolecules, 2004, 5, 326-333.

28 A. E. Donius, M. A. Kiechel, C. L. Schauer and U. G. K. Wegst, J. R. Soc. Interface, 2013, 10, 2012-0946.

29 J. D. Schiffman and C. L. Schauer, Biomacromolecules, 2007, 8, 59465601 .

30 T. Yoshihara, H. Tadokoro and S. Murahashi Journal of Chemical Physics, 1964, 41, 2902-2911.

31 Z. Osman and A.K. Arof Electrochimica Acta, 2003, 48, 993-999.
31 J. Xu, S.P. McCarthy, R.A. Gross and D.L. Kaplan, Macromolecules, 1996, 29, 3436-3440.

32 L.J. Bellamy, The Infrared Spectra of Complex Molecules, 1975, Chapman and Hall, New York

33 B.G. Johnansson, Scandinavian Journal of Clinical \& Laboratory Investigation, 1972, 29, 7-19.

7534 W. Samprasit, R. Kaomongkolgit, M. Sukma, T. Rojanarata, T. Ngawhirunpat and P. Opanasopit, Carbohydr. Polym., 2015, 117, $933-$ 940 .

35 Z. X. Meng, W. Zheng, L. Li, Y.F. Zheng, Mater. Chem. Phys., 2011, 125, 606-611. 